Practical Medicine, by Niemeyer, that he states on pp. 555 and 556: "During an epidemic (small-pox) persons who have been vaccinated but in whom the vaccination has not yet taken are attacked by small-pox and the two diseases run their course at the same time without modifying each other. These cases show that vaccination during the period of incubation of small-pox cannot cut short the disease and also that susceptibility to vaccination is not lost during the period of incubation." He goes on to say that vaccination and revaccination should be performed without delay as soon as small-pox is known in a neighbourhood. I am, Sirs, yours faithfully,

WM. BERRY. F.R C S.Irel., D.P.H.F.P.S.Glasg., Wigan, April 11th, 1898. Medical Officer of Health of Wigan.

\section{"AN UNKNOWN LARVAL PARASITE."} To the Editors of THE LANCET.

SIRs, -Cases similar to the one so ably described under the above heading ${ }^{1}$ by Dr. Frank Arnold are by no means rare on the West Coast of Lake Nyasa and in the country beyond. The worms are generally attributed by the natives to a fly of a mottled-brown colour, similar in shape to, but larger than, a big horse-fly, which is known by them as the "tshizeti." Some believe that the fly lays its eggs directly beneath the skin of the affected person; others that the eggs are deposited in large numbers upon blankets and other articles of clothing, and that the resultant maggots or "fusi" are able, while yet quite small, to penetrate the epidermis of the host.

Liverpool. I am, Sirs, yours faithfully,

StaNley KelletT Smith.

\section{NOTES FROM INDIA.}

\section{(From our Sphoial Correspondent.)}

Sudden Abandonment of Plague Operations in Bombay.-The

New Measures.-Increase of Plague in the Punjab.-The Wounded Europeans.

THe work of the Plague Committee in Bombay has for all practical purposes suddenly ceased. Not that the plague is decreasing-rather the reverse; but a new policy has been adopted by the Government. This change was contemplated before the riots on March $9 \mathrm{th}$, but a proclamation issued on March 15th states that general searching of houses for plague cases will be wholly given up. There will be no inspection of corpses and consequently no delay of funerals. The plague authorities will only take action against houses where undoubted cases of plague occur and there will be a relaxation of the pass systems, allowing free exit and ingress to bon $\hat{a}$ fide traders. This change is not only sudden but complete. It is stated that the operations of the Plague Committee were conducted in such a way as to create much discontent among, and hardships to, the native population, to very seriously hamper and injure the trade interests of Bombay, and to alienate all help from the natives themselves for the extermination of the disease. It is admitted that the results achieved have been far from satisfactory, but it is urged that a modification of the measures, which were based on general sanitary regulations, was all that was required. The new régime amounts to a distinct abdication of powers hitherto exercised by officers directly under Government to a collection of caste leaders with little or no sympathy in any sanitary measures for plague control, bearing little or no responsibility and undeterred by any consequences which may ensue. In trutb, however, there are no recognised native leaders and no native committees have been formed. It is now left to the natives themselves to take their sick to their own native hospitals if they choose, to report houses where cases of plague have occurred, also if they choose, and to vacate their houses and go into the health camps of their own accord. With the general dislike to hospitals it is almost certain that the sick will be left to die in their own homes. No kind of isolation whatsoever will be practised. The "contacts" will be free to move about undisturbed and a small proportion of infected tenements only

I The LaNCET, April 2nd, 1898, p. 960. will come under notice for disinfection. There will, in short, be no measures whatever adopted to check the spread of the contagion. From previous experience it is impossible to place any reliance whatever upon the natives themselves for following up any of the sanitary measures hitherto adopted for their benefit. Moreover, they have no organisation, and their efforts, even if reliable, are entirely voluntary. While the number of plague cases reported daily has probably fallen very far short of the correct number it is reasonable to assume that a much larger proportion will now fail to be registered. The total mortality will alone give any indication of the ravages of the disease. Whether or not other considerations have determined the adoption of this new policy it is almost certain that the results from a sanitary point of view must be disastrous.

The Europeans injured in the recent riots are all pro. gressing favourably. It is reported that a very much larger number of natives were killed and wounded than the figures officially published would seem to indicate. The plague is reported to be rapidly on the increase in the Punjab and many places in the Nizam's dominions have also been attacked. Preparations are being made to carry out Haffkine's prophylactic inoculations on a large scale in the latter territory. Among the 6000 Khojas of Bombay who have been inoculated only three cases of plague have occurred, which speaks highly for the protective value of this preparation. Some recent experiments with electrolysis of buboes have been attended with satisfactory results. Not only have the swellings rapidly diminished, but almost immediate improvement has been recognised in the general symptoms. From 10 to 15 milliampères is the strength used, which is kept up for ten minutes, and in most of the cases one operation has sufficed. This treatment is still being followed up and some results will be able to be published shortly.

The modified measures recently adopted by the Government appeared at first sight to have appeased popular feeling, bat there are evidences of considerable discontent with even the very mild steps now taken. The following rules just promulgated contrast very strongly with the regular house-to-house visitation which was formerly carried out together with the compulsory segregation of all cases found into hospital, of many of the contacts to the observation camps, the emptying of crowded and insanitary houses, and the disinfection of plague-stricken premises on a large scale. It is now ordered that house-to-house visitation be carried on by the people themselves-i.e., by committees appointed from among them. Plague officials will search only those houses where cases or deaths have recently occurred or where there is a reasonable suspicion of a plagne case.

No patient will be removed to a plague hospital unless an approved and fully qualified medical officer certifies it to be a plague case. If such a medical officer pronounces a. case to be hopeless-i.e., only expected to live a few hourssuch patient will not be removed to a hospital without the consent of the relatives. In the erent of a case of plague occurring in the family of a well-known and well-to-do or respectable person the patient will be allowed to remain in the house and can be attended there by his own doctor and by one or two members of his family, provided the house is a detached one and the patient can be isolated and the family agree to remove themselves to one of the Plague Committee's health camps, or to dwell at a detached place where they can be placed under supervision. Where disinfection will suffice no destruction of property is now allowed. If, to diminish risk of infection, destruction of property is found to be necessary, compensation will, in the case of the poor, be paid on the spot. Infected houses will continue to be disinfected as heretofore. The owners or occupiers will be given the option of disinfecting their houses themselves to the satisfaction of the district plague officer. Contacts will continue to be removed as before.

The policy of depending upon the natives to notify cases of plague has not yet shown any improvement in the number detected. It is estimated that 600 cases of plague occur weekly which escape discovery by the staff of the Plague Committee. This is about half the mortality from plague. Large numbers die in their own homes, and large numbers are taken to the hospitals in the last stage of the disease and die there within a few hours. There is no reduction in the general mortality, and plague has now gradually spread to every quarter of the city in a very similar manner to what was observed last year. Fresh orders, still further in the 